\title{
Usefulness of palliative prognostic score in the treatment of patients with non-resectable gastric cancer
}

\author{
MASAHIDE IKEGUCHI, ABDUL KADER, MIWA YOSHIMOTO, SEIGO TAKAYA, JOJI WATANABE, \\ YOUJI FUKUMOTO, TOMOHIRO OSAKI, HIROAKI SAITO, SHIGERU TATEBE and TOSHIRO WAKATSUKI \\ Department of Surgery, Division of Surgical Oncology, Faculty of Medicine, Tottori University, Yonago 683-8504, Japan
}

Received July 11, 2012; Accepted December 11, 2012

DOI: $10.3892 / \mathrm{mco} .2013 .66$

\begin{abstract}
The aim of this study was to evaluate the clinical usefulness of the palliative prognostic $(\mathrm{PaP})$ score in patients with non-resectable advanced gastric cancer. The PaP score was calculated prior to each course of chemotherapy in 44 consecutive patients with non-resectable advanced gastric cancer between 2003 and 2010 at the Tottori University Hospital, Yonago, Japan. The prognosis was evaluated according to the PaP score and the different chemotherapeutic agents. The median survival time (MST) was 10 months. The $\mathrm{PaP}$ score classified the heterogeneous patient sample into three isoprognostic groups with regard to the possibility of a 1-month survival period, with 28 patients in group A ( $>70 \%$ chance), 12 in group B (30-70\% chance) and 4 in group $C$ ( $<30 \%$ chance). The MST of the three groups was 11, 3 and 1 months for group $\mathrm{A}, \mathrm{B}$ and $\mathrm{C}$, respectively. In group A, chemotherapeutic regimens did not affect patient survival, although the docetaxel regimen prolonged survival of patients in group B. In conclusion, the PaP score may be useful in selecting the best chemotherapeutic regimen in patients with non-resectable gastric cancer.
\end{abstract}

\section{Introduction}

Outcomes are extremely poor in patients with non-resectable gastric cancer, with a median survival period ranging from 3 to 5 months, even with the best supportive care $(1,2)$. S-1 is an oral anticancer drug that combines tegafur, a prodrug of fluorouracil, with 5-chloro-2,4-dihydropyrimidine (CDHP) and potassium oxonate at a molar ratio of 1:0.4:1 (Taiho Pharmaceutical Co., Ltd., Tokyo, Japan) (3). In a phase II study of S-1, an $\sim 40 \%$ response rate was noted in patients with advanced gastric cancer $(4,5)$. Thus, S-1 chemotherapy has been widely used as a basic treatment for patients with

Correspondence to: Dr Masahide Ikeguchi, Department of Surgery, Division of Surgical Oncology, Faculty of Medicine, Tottori University, 36-1 Nishi-cho, Yonago 683-8504, Japan

E-mail: masaike@med.tottori-u.ac.jp

Key words: docetaxel, palliative prognostic score, non-resectable gastric cancer non-resectable gastric cancer. Findings from the SPIRIT trial identified S-1 plus cisplatin as a standard first-line treatment (6) and recommended its use in patients with an expected survival period of at least 3 months. However, due to the severe side effects, the $\mathrm{S}-1$ plus cisplatin regimen $\left[\mathrm{S}-1: 40-60 \mathrm{mg} / \mathrm{m}^{2}\right.$; in a 5 -week cycle ( 3 weeks on and 2 weeks off), in combination with $60 \mathrm{mg} / \mathrm{m}^{2}$ cisplatin on day 8] was difficult to continue in patients with poor Eastern Cooperative Oncology Group Performance Status (ECOG PS). Additionally, a number of patients suffered from reduced quality of life (QOL) while undergoing this medical treatment (7). However, Casaretto et al (8) reported that chemotherapy increased the 1-year survival rate, provided a longer symptom-free period and improved the QOL of patients with non-resectable advanced gastric cancer. Clinically, it is important to select chemotherapeutic regimens that are most appropriate for the patient's condition.

The objective indicators determining suitable chemotherapy regimens for patients with non-resectable gastric cancer have been studied. The standard prognostic indicators in oncology, such as tumor size, grade and stage, or molecular biology, are less relevant in patients with advanced cancer. The palliative prognostic $(\mathrm{PaP})$ score was developed in the 1990 s, as a result of a series of prospective trials aimed to identify clinical and biologic factors associated with the prognosis of advanced cancer patients referred to hospice and to merge them into a prognostic index (9). The survival of patients with non-resectable or recurrent cancers can be estimated using the PaP score even during chemotherapy (10).

In this study, the usefulness of the PaP score in determining the first-line chemotherapy for patients with non-resectable gastric cancer was examined retrospectively.

\section{Materials and methods}

Patients. Between 2003 and 2010, 558 patients with gastric cancer were treated at the Tottori University Hospital, Yonago, Japan. Forty-four patients (7.9\%) were diagnosed as non-resectable. Details of these 44 patients are shown in Table I. Patients were followed up at the hospital until March 2012. During this period, gastrectomy was performed on 3 patients (bleeding, 2 patients; perforation, 1 patient). All participants provided informed consent and the study design was approved by the Ethics Review Board of Tottori University. 
Table I. Patient data $(n=44)$.

\begin{tabular}{lc}
\hline Variables & No. \\
\hline Age (range, mean; years) & $23-92,66.5$ \\
Gender (male/female) & $24 / 20$ \\
Ascites (yes/no) & $10 / 34$ \\
ECOG PS (0/1/2) & $14 / 18 / 12$ \\
Non-resectable parameters & \\
Locally advanced & 6 \\
Lymph node & 12 \\
Hematogenic metastasis & 19 \\
Peritoneal metastasis & 20 \\
Surgical intervention & \\
No & 29 \\
Probe-laparotomy & 1 \\
Bypass operation & 11 \\
Gastrectomy & 3 \\
\hline
\end{tabular}

ECOG PS, Eastern Cooperative Oncology Group performance status.

Chemotherapy. First-line chemotherapy was received by 41 patients (S-1, 7; S-1 plus cisplatin, 17; S-1 plus docetaxel, 13; other chemotherapy, 4). Chemotherapy was terminated in the case of 3 patients with poor performance status (PS) and advanced age, who then received best supportive care (BSC).

PaP score. The PaP score has four criteria: two symptoms (anorexia and dyspnea), performance status measured by the Karnofsky performance score, white blood cells (WBC) abnormalities (high total WBC count and lymphopenia) and a physician's survival prediction measured in weeks (Table II). Validated cut-off points based on the total PaP score were established to classify the patients into three prognostic groups for survival at 30 days: group A ( $>70 \%$ probability of a 1-month survival period), 0 to 5.5 points; group B (30-70\% probability of a 1-month survival period), 5.6 to 11 points; group $\mathrm{C}$ (<30\% probability of a 1-month survival), 11.1-17.5 points $(10,11)$ (Table II).

Statistical analysis. The terminology used in this study conforms to the Japanese Classification of Gastric Carcinoma, 3rd English edition (12). Statistical analysis was carried out using $\chi^{2}$ tests. Overall survival was calculated from the time of enrolment to death. Median survival time (MST) was calculated using the Kaplan-Meier non-parametric test, while comparison between the different patient cohorts was performed using the log-rank test. $\mathrm{P}<0.05$ was considered to indicate a statistically significant difference.

\section{Results}

Median survival time. The MST of the 44 patients was 10 months. Patients were divided into 3 subgroups, according to their PaP score. The MST of 28 patients in group A
Table II. PaP score.

Item Score

Symptoms (presence/absence)

Anorexia $\quad 1.0 / 0.0$

Dyspnea $\quad 1.5 / 0.0$

Karnofsky performance status

$\geq 50 \quad 0.0$

$30-40 \quad 0.0$

$10-20 \quad 2.5$

Clinical prediction of survival (weeks)

$>12$

$11-12 \quad 2.0$

9-10 2.5

$7-8 \quad 2.5$

$5-6 \quad 4.5$

3-4 6.0

$1-2 \quad 8.5$

Total white blood cells $\left(/ \mathrm{mm}^{3}\right)$

Normal $(4,800-8,500)$

High $(8,501-11,000) \quad 0.5$

Very high $(>11,000) \quad 1.5$

Lymphocyte percentage

Normal (20.0-40.0) 0

Low (12.0-19.9) 1.0

Very low (0-11.9) 2.5

PaP score groups

A

B

5.6-11.0

$\mathrm{C}$

$11.1-17.5$

(11 months) was much better compared to the 12 patients in group B (3 months) or the 4 patients in group C (1 month, $\mathrm{P}<0.0001$, Fig. 1). In the 40 patients in groups $\mathrm{A}$ and $\mathrm{B}$, the correlation between prognosis and factors considered to affect the prognosis was analyzed (Table III). The presence or absence of ascites or bypass surgery did not affect patient survival.

Correlation between the PaP score and the first-line chemotherapy regimens. The correlation between the $\mathrm{PaP}$ score and the first-line chemotherapy regimens are shown in Table IV. The S-1 plus cisplatin regimen was commonly used as firstline chemotherapy in PaP group A. However, due to renal dysfunction, cisplatin was not used in a number of patients in group B, thus S-1 plus docetaxel or S-1 alone was selected in this group instead. In the 28 patients in group A, the MST using the cisplatin regimen (10 months, $n=16)$ did not differ from the other regimens ( 11 months, $\mathrm{n}=12, \mathrm{P}=0.221)$. Although the difference was not significant $(\mathrm{P}=0.062)$, in the 12 patients 
Table III. Prognosis of patients in PaP score groups A and B.

\begin{tabular}{lrcc}
\hline & N & MST (months) & P-value \\
\hline PaP score group & & & \\
A & 28 & 11 & 0.0045 \\
B & 12 & 3 & \\
Ascites & & & \\
Absent & 31 & 10 & 0.7548 \\
Present & 9 & 10 & \\
Surgical intervention & & & 0.7238 \\
No & 25 & 10 & \\
Yes & 15 & 11 & \\
\hline
\end{tabular}

PaP, palliative prognostic; MST, median survival time.

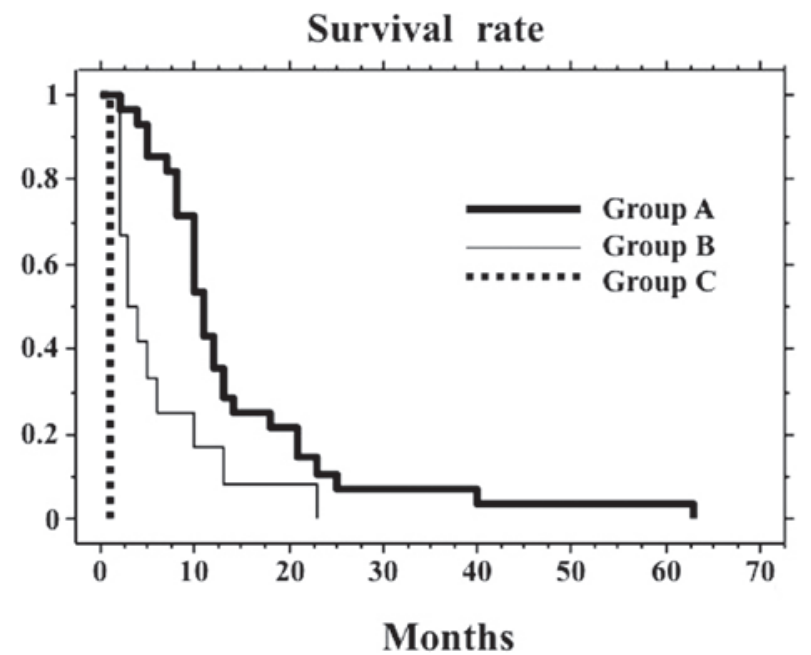

Figure 1. Survival of patients in group A was much better compared to patients in group $\mathrm{B}$ or $\mathrm{C}(\mathrm{P}<0.0001)$.

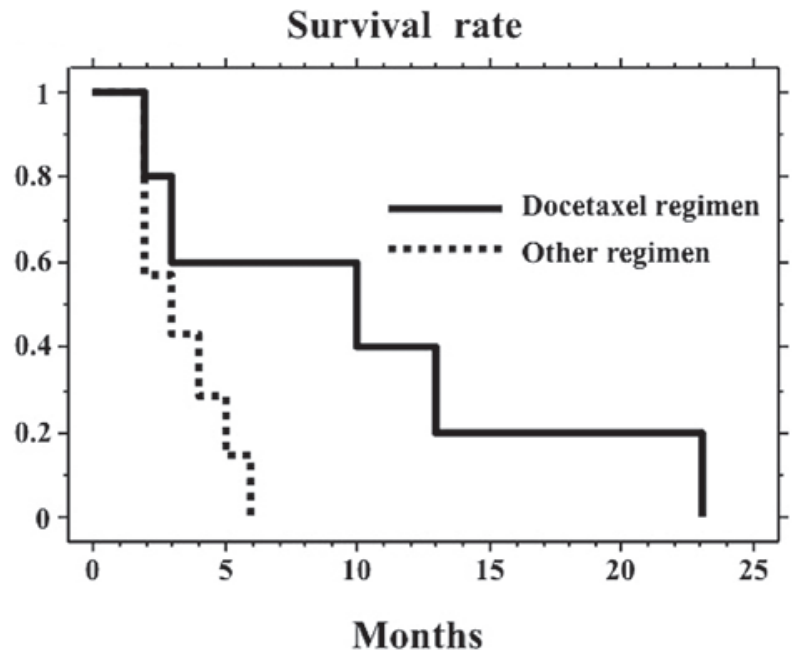

Figure 2. Survival of the 5 patients treated with docetaxel was better compared to the 7 patients treated with other regimens in the PaP group B, with no statistically significant difference $(\mathrm{P}=0.062)$.
Table IV. Correlation between PaP score and first-line chemotherapy.

\begin{tabular}{lrrr}
\hline & \multicolumn{3}{c}{ PaP score group } \\
\cline { 2 - 4 } First-line chemotherapy & A & B & C \\
\hline S-1 plus cisplatin & 16 & 1 & 0 \\
S-1 plus docetaxel & 8 & 5 & 0 \\
S-1 plus CPT-11 & 2 & 2 & 0 \\
S-1 only & 2 & 4 & 1 \\
BSC & 0 & 0 & 3 \\
\hline
\end{tabular}

PaP, palliative prognostic; BSC, best supportive care.

in group $\mathrm{B}$, the docetaxel regimen prolonged the survival from 3 (other regimens, MST, 3 months, n=7) to 10 months (docetaxel regimen, MST, 10 months, n=5, Fig. 2).

\section{Discussion}

Large-scale randomized phase III clinical trials may reveal effective chemotherapeutic regimens for patients with advanced cancers, with the exception of those of advanced age or with poor PS. However, in clinical situations, it is difficult to decide the most suitable chemotherapeutic regimen for patients with short life expectancy or poor PS, such as patients with non-resectable gastric cancer. Identifying the patients that may benefit from palliative chemotherapy is quite difficult and its usefulness when controlling symptoms and maintaining QOL has not yet been proven $(13,14)$.

The PaP score contains five parameters (symptom, PS, inflammation, immunity and physician's survival prediction) associated with cancer patient survival. Findings of previous studies have indicated that the PaP score may accurately estimate pre-terminal patient survival (15-17). Using the PaP score in 44 patients with non-resectable advanced gastric cancer, the correlation between PaP score groups and chemotherapeutic regimens was investigated. The findings showed that in the PaP score group $\mathrm{A}$, the $\mathrm{S}-1$ plus cisplatin regimen was commonly used and differences in chemotherapeutic regimens did not affect the survival of the patients in this group. In comparison, the survival of patients in $\mathrm{PaP}$ group $\mathrm{B}$ was extremely poor, although the S-1 plus docetaxel regimen prolonged the survival of these patients from 3 to 10 months. Although this study is retrospective and the number of objective cases is small, the docetaxel regimen may have a survival advantage in patients with a poor prognosis.

Docetaxel is reported to have a low rate of grade $3 / 4$ leucopenia and neutropenia (19.4 and 10.6\%) and rare, severe non-hematologic toxicities (18). Docetaxel chemotherapy with or without S-1 has been a suitable treatment for patients with advanced gastric cancer, advanced age or poor PS (19).

In conclusion, in the treatment of advanced non-resectable gastric cancer, the $\mathrm{PaP}$ score should be used to select patients and chemotherapeutic regimens. The S-1 plus docetaxel regimen is expected to improve outcomes in patients with a poor PS. 


\section{References}

1. Murad AM, Santiago FF, Petroianu A, Rocha PR, Rodrigues MA and Rausch M: Modified therapy with 5-fluorouracil, doxorubicin, and methotrexate in advanced gastric cancer. Cancer 72: 37-41, 1993.

2. Glimelius B, Hoffman K, Haglund U, Nyren O and Sjoden PO: Initial or delayed chemotherapy with best supportive care in advanced gastric cancer. Ann Oncol 5: 189-190, 1994.

3. Shirasaka T, Shimamato Y, Ohshima H, Yamaguchi M, Kato T, Yonekura K and Fukushima M: Development of a novel form of an oral 5-fluorouracil derivative (S-1) directed to the potentiation of the tumor selective cytotoxicity of 5-fluorouracil by two biochemical modulators. Anticancer Drugs 7: 548-557, 1996.

4. Sakata Y, Ohtsu A, Horikoshi N, Sugimachi K, Mitachi Y and Taguchi T: Late phase II study of novel oral fluoropyrimidine anticancer drug S-1 (1 M tegafur- $0.4 \mathrm{M}$ gimestat- $1 \mathrm{M}$ otastat potassium) in advanced gastric cancer patients. Eur J Cancer 34: 1715-1720, 1998.

5. Koizumi W, Kurihara M, Nakano S and Hasegawa K: Phase II study of S-1, a novel oral derivative of 5-fluorouracil, in advanced gastric cancer. For the S-1 Cooperative Gastric Cancer Study Group. Oncology 58: 191-197, 2000

6. Koizumi W, Narahara H, Hara T, Takagane A, Akiya T, Takagi M, Miyashita K, Nishizaki T, Kobayashi O, Takiyama W, Toh Y, Nagaie T, Takagi S, Yamamura Y, Yanaoka K, Orita H and Takeuchi M: S-1 plus cisplatin versus S-1 alone for first-line treatment of advanced gastric cancer (SPIRITS trial): a phase III trial. Lancet Oncol 9: 215-221, 2008.

7. Koo DH, Ryu MH, Ryoo BY, Lee SS, Moon JH, Chang HM, Lee JL, Kim TW and Kang YK: Three-week combination chemotherapy with S-1 and cisplatin as first-line treatment in patients with advanced gastric cancer: a retrospective study with 159 patients. Gastric Cancer 15: 305-312, 2012.

8. Casaretto L, Sousa PLR and Mari JJ: Chemotherapy versus support cancer treatment in advanced gastric cancer: a metaanalysis. Braz J Med Biol Res 39: 431-440, 2006.

9. Pirovano M, Maltoni M, Nanni O, Marinari M, Indelli M, Zaninetta G, Petrella V, Bami S, Zecca E, Scarpi E, Labianca R, Amadori D and Luporini G: A new palliative prognostic score: A first step for the staging of terminally ill cancer patients. J Pain Symptom Manage 17: 231-239, 1999.
10. Tassinari D, Montanari L, Maltoni M, Ballardini M, Piancastelli A, Musi M, Porzio G, Minotti V, Caraceni A, Poggi B, Stella A, Aielli F and Scarpi E: The palliative prognostic score and survival in patients with advanced solid tumors receiving chemotherapy. Support Care Cancer 16: 359-370, 2008.

11. Maltoni M, Nanni O, Pirovano M, Scarpi E, Indelli M, Martini C, Monti M, Amoldi E, Piva L, Ravaioli A, Cruciani G, Labianca R and Amadori D: Successful validation of the palliative prognostic score in terminally ill cancer patients. J Pain Symptom Manage 17: 240-247, 1999.

12. Japanese Gastric Cancer Association. Japanese classification of gastric carcinoma. 3rd English edition. Gastric Cancer 14: 101-112, 2011.

13. Geels P, Eisenhauer E, Bezjak A, Zee B and Day A: Palliative effect of chemotherapy: objective tumor response is associated with symptom improvement in patients with metastatic breast cancer. J Clin Oncol 18: 2395-2405, 2000.

14. Browner I and Carducci MA: Palliative chemotherapy: historical perspective, applications and controversies. Semin Oncol 32: 145-155, 2005.

15. Glare PA, Eychmueller S and McMahon P: Diagnostic accuracy of the palliative prognostic score in hospitalized patients with advanced cancer. J Clin Oncol 22: 4823-4828, 2004.

16. Stone CA, Tiernan E and Dooley BA: Prospective validation of the palliative prognostic index in patients with cancer. J Pain Symptom Manage 35: 617-622, 2008.

17. Stiel S, Bertram L, Neuhaus S, Nauck F, Ostgathe C, Elsner F and Radbruch L: Evaluation and comparison of two prognostic scores and the physicians' estimate of survival in terminally ill patients. Support Care Cancer 18: 43-49, 2010.

18. Massacesi C, Marcucci F, Rocchi MB, Mazzanti P, Pilone A and Bonsignori M: Factors predicting docetaxel-related toxicity: experience at a single institution. J Chemother 16: 86-93, 2004

19. Fujii M: Chemotherapy for advanced gastric cancer: ongoing phase III study of S-1 alone versus S-1 and docetaxel combination (JACCRO GC03 study). Int J Clin Oncol 13: 201-205, 2008. 